\title{
Characterization of a Fungal Thioesterase Having Claisen Cyclase and Deacetylase Activities in Melanin Biosynthesis
}

\author{
Anna L Vagstad ${ }^{1}$, Eric A Hill ${ }^{1}$, Jason W Labonte ${ }^{1}$, and Craig A Townsend ${ }^{1,}$, \\ ${ }^{1}$ Department of Chemistry, The Johns Hopkins University, Baltimore, Maryland 21218, USA
}

\section{Summary}

\begin{abstract}
Melanins are a broad class of darkly-pigmented macromolecules formed by oxidative polymerization of phenolic monomers. In fungi, melanins are known virulence factors that contribute to pathogenicity. Their biosynthesis generally involves polymerization of 1,8 dihydroxynaphthalene via a 1,3,6,8- tetrahydroxynaphthalene (THN) precursor assembled by multidomain, nonreducing polyketide synthases. Multiple, convergent routes to THN have evolved in fungi. Parallel heptaketide and hexaketide pathways exist that utilize conventional Cterminal thioesterase/Claisen cyclase domains and separate side-chain deacylases. Here, in vitro characterization of Pks1 from Colletotrichum lagenarium establishes a true THN synthase with a bifunctional thioesterase (TE) catalyzing both cyclization and deacetylation of an enzyme-bound hexaketide substrate. Chimeric TE domains were generated by swapping lid regions of active sites between classes of melanin TEs to gain insight into this unprecedented catalysis of carbon-carbon bond making and breaking by an $\alpha / \beta$-hydrolase fold enzyme.
\end{abstract}

\section{Introduction}

Fungal infections afflict millions of people and cause crop losses in the $\$$ billions each year (Gladieux, et al., 2011). Many human pathogenic fungi are dematiaceous yeasts that produce dark cell-wall pigments called melanins and are among the most resistant to current antifungal treatments (Nosanchuk and Casadevall, 2006). Melanin is linked to fungal virulence in a variety of hosts. Human pathogens with melanized cell walls are more resistant to damage from the host immune response, promoting, for example, resistance to phagocytosis (Thywißen, et al., 2011) and reactive oxygen species (Cunha, et al., 2010) mustered in self-defense by macrophages. Inhibition of melanin production has been proposed as an effective route to controlling fungal infections in susceptible immunecompromised populations (Liu and Nizet, 2009). In phytopathogenic fungi, melanin biosynthetic enzymes are validated targets for fungicides, which are widely used to prevent crop damage (Wheeler and Klich, 1995). Understanding the fundamental principles of melanogenesis may enable development of new antifungals to combat infections.

The major pathway for melanin biosynthesis in fungi is through polymerization of polyketide-derived 1,8-dihydroxynaphthalene (DHN, 1) monomers into dark brown or black cell-wall pigments. Melanins are highly insoluble and thus resistant to structural

\footnotetext{
(C) 2012 Elsevier Ltd. All rights reserved.

*Corresponding author: Prof. Craig A. Townsend, Department of Chemistry, Remsen Hall 252, 3400 N. Charles Street, Baltimore MD 21218-2685, PHONE (410) 516-7444, FAX (410) 261-1233, ctownsend@ jhu.edu.

Publisher's Disclaimer: This is a PDF file of an unedited manuscript that has been accepted for publication. As a service to our customers we are providing this early version of the manuscript. The manuscript will undergo copyediting, typesetting, and review of the resulting proof before it is published in its final citable form. Please note that during the production process errors may be discovered which could affect the content, and all legal disclaimers that apply to the journal pertain.
} 
characterization, but have electrochemical and mechanical properties that help exert their protective or virulent functions (Jacobson, 2000). The pivotal intermediate of the biosynthetic pathways is 1,3,6,8-tetrahydroxynaphthalene (THN, 2), which undergoes two rounds of enzymatic reduction and dehydration to 1 . The THN skeleton is synthesized by multidomain nonreducing iterative polyketide synthases (NR-PKSs). In some cases, melanin genes are clustered at one genetic locus, but they can also be dispersed throughout the genome (Woo, et al., 2010). Although nonessential, melanin pigments are important for survival and longevity. In many opportunistic plant and human pathogenic fungi, colorblock mutants are significantly less or not infective (Bell and Wheeler, 1986; Langfelder, et al., 2003). Colletotrichum lagenarium (syn. C. orbiculare) is a dematiaceous fungus that infects members of the cucumber family and causes anthracnose disease (Tsuji, et al., 2003). Melanin structurally stabilizes the cell wall, facilitating turgor pressure to build up in specialized infectious bodies called appressoria, and is essential for host penetration (Pihet, et al., 2009; Takano, et al., 1997). Treatment of $C$. lagenarium with the melanin inhibitor tricyclazole (which targets THN reductase) induces an albino phenotype, causes flaviolin (3, the autoxidation product of THN) to accumulate, and prevents infiltration of the plant dermis (Kubo, et al., 1985).

The importance of THN (2) is highlighted by the existence of convergent biosynthetic routes to this common metabolite in different fungi, as outlined in Figure 1. In Aspergillus fumigatus, heptaketide YWA1 (4) is the hemiketal of the direct product of the Alb1 NRPKS (Watanabe, et al., 2000). The acetoacetyl side chain is removed by a separate serine hydrolase, Ayg1p, to yield THN (Tsai, et al., 2001). In an alternative pathway, WdPks1 from zoopathogenic Wangiella (Exophiala) dermatitidis synthesizes a hexaketide, 2acetyl-1,3,6,8-tetrahydroxynaphthalene (ATHN, 5), and the acetyl side chain is likewise removed by WdYg1p, an Ayg1p homolog (Wheeler, et al., 2008). A handful of examples of naphtho- $\gamma$-pyrone synthases have been biochemically characterized in other fungi with wA from $A$. nidulans being a paradigm example. wA synthesizes YWA1, which is thought to be polymerized directly into melanin-related conidial green pigment, circumventing the THN intermediate (Watanabe, et al., 1999). These fungal pathways are distinct from bacterial type III THN synthases, such as RppA from Streptomyces griseus, where five malonyl units are condensed to directly form the pentaketide as a precursor in hexahydroxyperylenequinone melanin biosynthesis (Funa, et al., 2005; Funa, et al., 2002).

Fungal NR-PKSs are a family of multidomain enzymes that generate substituted aromatic natural products. Structural diversity is achieved through "programmed" selection of starter unit, polyketide chain length, regiospecificity of backbone cyclization(s), and mode of product release. Enormous progress has been made in the last decade to understand the catalytic function of the six canonical domains that comprise these enzymes and how they orchestrate polyketide assembly (Crawford and Townsend, 2010). The $\beta$-ketoacyl synthase (KS), malonyl-CoA: acyl-carrier protein transacylase (MAT), and acyl-carrier protein (ACP) were functionally identified by their similarity to domains from fatty acid metabolism. Like fatty acid biosynthesis, each round of iterative extension introduces a single $\mathrm{C}_{2}$-unit through decarboxylative thio-Claisen condensation of malonyl with the extending chain. Intermediates are tethered as terminal thioesters of a $4^{\prime}$-phosphopantetheinyl moiety attached to the ACP. The N-terminal domain, dubbed the starter unit: acyl-carrier protein transacylase (SAT), initiates synthesis with a prescribed starter unit, typically acetyl from the acyl-CoA (Crawford, et al., 2006; Crawford, et al., 2008). The product template domain (PT) catalyzes regiospecific cyclization and aromatization of the nascent ACP-bound $\beta$-keto chain (Crawford, et al., 2009; Li, et al., 2010). In melanin-type NR-PKSs connectivity between the $\mathrm{C} 2$ and $\mathrm{C} 7$ backbone carbons produces a monocyclic intermediate, as shown in Figure 1. The C-terminus constitutes a thioesterase domain (TE) responsible for product release. The Claisen/Dieckmann cyclase class of TE domains (TE/CLC), which catalyzes C- 
$\mathrm{C}$ bond formation in contrast to the classical hydrolytic release of fatty acids, was originally identified in the wA naphthopyrone synthase from A. nidulans (Fujii, et al., 2001). Further insight into this class of TEs was advanced through determination of the crystal structure of a dissected TE/CLC monodomain from the norsolorinic acid anthrone synthase, PksA, from A. parasiticus (Korman, et al., 2010). Inactivating mutation or deletion of TE/CLC domains generally results in spontaneous $\mathrm{O}-\mathrm{C}$ cyclization to release full-length pyrone shunt products (Crawford and Townsend, 2010).

Historically, metabolites originating from NR-PKSs fit this model of polyketide assembly; however, Pks1 from $C$. lagenarium appeared to be an outlier. THN (2) was identified as the major product from cultures of Pks1 heterologously expressed in A. oryzae (Fujii, et al., 1999). Application of ${ }^{14} \mathrm{C}$-labeled acetyl or malonyl substrates showed no apparent incorporation of an acetyl starter unit and that pentaketide $\mathbf{2}$ was produced solely from malonyl units (Fujii, et al., 2000). When the TE domain was inactivated, hexaketide pyrone 7 was produced instead, paralleling the synthetic logic since shown by other NR-PKS systems (Crawford, et al., 2008; Zhang, et al., 2008). Furthermore, expression of a chimera that spliced Pks1 to the C-terminus of the wA gene at the PT-ACP junction resulted in isolation of the nonnative hexaketide Claisen cyclase product, ATHN (5) (Watanabe and Ebizuka, 2002). Based on the prevailing understanding of PKS catalysis at the time of this publication, the ability to select for the pentaketide chain length was ascribed to a special function of the Pks1 TE domain. Subsequent experiments with NR-PKS family members, however, support the view that chain length is determined by the KS domain, making this "TE chain length control" theory untenable for Pks1 (Crawford and Townsend, 2010).

In corresponding studies, Ayg $1 \mathrm{p}$ was identified as the side-chain hydrolase that removes acetoacetyl from YWA1 in A. fumigatus (Tsai, et al., 2001). A BlastP search of the sequenced A. oryzae genome (Machida, et al., 2005) for proteins similar to Ayg 1p revealed a potential functional complement in this heterologous host, the transcription of which was supported under standard laboratory conditions by detection of an expressed sequence tag (AoEST6890) from a conidial cDNA library (Akao, et al., 2007). Furthermore, the purified Pks1 SAT monodomain was found to selectively transfer acetyl in disparity with labeledprecursor incorporation experiments (Crawford, et al., 2008). Taking into account these results and the precedence of fungal NR-PKSs catalyzing chain extension prior to cyclization in the PT and TE domains, we hypothesized that $C$. lagenarium (and heterologous host $A$. oryzae) contained an enzyme with Ayg1p-like activity and that hexaketide ATHN (5) was the direct product of Pks1 (Crawford, et al., 2008). Such a hexaketide precursor was originally proposed by Bardshiri and Simpson when feeding experiments with labeled acetate failed to reveal a "starter-unit effect" for melanin intermediates isolated from Phialaphora lagerbergii (Bardshiri and Simpson, 1983). However, the supposition of an Ayg1p-like activity in A. oryzae was undermined when heterologous expression of the melanin PKS from $W$. dermatitidis (WdPks1) in this host accumulated ATHN (Wheeler, et al., 2008), not THN as had been observed for expression of Pks1 under similar conditions. These observations led us to suspect that something else was occurring. We chose, therefore, to study Pks1 in vitro to establish the biochemical rationale for THN production in C. lagenarium.

\section{Results}

\section{Synthesis of Standards}

To proceed with this study, synthetic standards of melanin biosynthetic intermediates and shunt metabolites were prepared to confidently identify the products of in vitro Pks1 reactions. Most of the synthetic methods utilized were standard procedures, as detailed in Figure 2 and the Supplemental Information. The synthesis of ATHN (5, Figure 2) initially 
proved troublesome, but was efficiently achieved by way of double $O$-acyclation/Fries rearrangements to generate the asymmetric penta-substituted phenyl ester $\mathbf{8}$.

Methoxymethyl protection of the ester $\mathbf{9}$ followed by coupling with the Weinreb amide $\mathbf{1 0}$ gave the ketone 11. Enolate cyclization, removal of the silyl group, and oxidation led to the desired hexaketide pyrone $\mathbf{7}$ after removal of the methoxymethyl groups. Dibenzyl protection of the ester $\mathbf{1 2}$ followed by Friedel-Crafts acylation afforded the acetophenone $\mathbf{1 3}$. Treatment with methoxide in methanol yielded dibenzyl THN (14), which was deprotected by hydrogenolysis immediately before use for HPLC analysis of THN (2) or readily oxidized in air to flaviolin (3). The double $O$-acylation/Fries rearrangements noted above, selectively afforded the asymmetric penta-substituted phenyl ester $\mathbf{8}$. Di- $O$-benzyl protection and Dieckmann condensation generated 3-di-O-benzyl ATHN, which following hydrogenolysis gave ATHN (5).

\section{Protein Deconstruction and Correction of the Pks1 Coding Sequence}

To study NR-PKSs in vitro, we have adopted a "deconstruction" approach based on in silico prediction of domain boundaries to dissect expressible, functional units from target enzymes (Udwary, et al., 2002). This approach offers a versatile method to assess domains individually or in selected sets, as exemplified by the four-part reconstitution of $A$. parasiticus PksA activity to synthesize norsolorinic acid anthrone, the first committed intermediate in aflatoxin biosynthesis (Crawford, et al., 2008). Expression plasmids to study melanin biosynthesis by Pks1 from C. lagenarium were patterned on analogous interdomain cleavage sites used for deconstruction of PksA. Soluble, active proteins were readily achieved for all constructs, except those containing the TE domain-a domain typically overexpressed without difficulty.

The current annotation for Pks1 (GenBank accession BAA18956) designates two short exons within the $\mathrm{N}$-terminal SAT domain and a third long exon spanning all domains. The Pks1 protein sequence was aligned to other characterized NR-PKSs for comparative analysis. Close inspection revealed that all catalytic residues and secondary structural elements appeared to be intact. Despite its correct appearance, re-assessment of Pks1 gDNA using FGNESH gene prediction software (softberry.com) exposed an alternative protein sequence where the final 17 residues of exon 3 were replaced by a short 14-amino-acid exon 4. Constructs based on this modified cDNA resulted in soluble expression of the functional TE domain. Furthermore, transcript analysis of the region straddling the in silico-predicted pks1 exon 3-4 junction confirmed that this revised splicing pattern is observed in $C$. lagenarium mRNA, in amendment of the original gene structure report (Takano, et al., 1995). In the first study of Pks1 activity, the cDNA clone used for heterologous expression in A. oryzae was based on the original three-exon gene structure, but additionally contained extra genomic sequence at its $3^{\prime}$ terminus that encompassed the proposed fourth exon (Fujii, et al., 1999). The heterologous host $A$. oryzae was able to complement native mRNA splicing to afford the active enzyme. Additional transcript evidence in support of a revised gene structure was identified in the melanin PKS (GenBank accession CCF45141) from the closely related species $C$. higginsianum (Ushimaru, et al., 2010), which has a TE domain that shares $94.4 \%$ protein sequence identity with Pks1. Expressed sequence tags reported for ChPks1 (CH063_03518, Broad Institute) in the Colletotrichum genome database confirm that the $C$. higginsianum gene transcript contains the same four-exon structure validated here for C. lagenarium Pks1.

\section{Domain Swapping Reveals a Thioesterase with Claisen Cyclase and Deacetylase Activities}

The second advantage of the deconstruction approach is that individually expressed domains can be "mixed and matched," offering a flexible platform for domain swapping among 
different NR-PKSs. In an initial set of reactions, Pks1 SAT-KS-MAT-PT-ACP-ACP (Pks1 $\triangle \mathrm{TE}$ ) was combined with either the Pks1 or wA TE monodomain. Reaction progress was monitored by UV-vis spectrophotometry and products were separated by reverse-phase HPLC and identified by comparison to synthetic standards (Figure 3). The Pks1 TE reaction rapidly accumulated an absorbance peak at $336 \mathrm{~nm}$, indicative of THN production (not ATHN as predicted if a second Ayg1p-like enzyme were invoked). THN (2) spontaneously converts to the reddish-purple oxidation product flaviolin (3), the major species observed by HPLC. Trace amounts of $\mathrm{C}_{12}$-pyrone 7 and ATHN (5) were also detected. In contrast, the wA TE reaction developed a $\lambda_{\max }$ at $411 \mathrm{~nm}$, producing mainly 5 to mirror the product profile observed previously for the full length Pks1-wA chimera expressed in A. oryzae (Watanabe and Ebizuka, 2002). ATHN spontaneously oxidizes to 3-acetyl-flaviolin (3-AF, 15) over time, albeit at a slower rate than THN oxidation (Wheeler, et al., 2008). The control reaction with no TE gradually accumulated 7 , formed by spontaneous $\mathrm{O}-\mathrm{C}$ cyclization of the monocyclic ACP-bound product of the PT domain.

The complementary experiment was conducted using the deconstructed SAT-KS-MAT and PT-ACP-ACP proteins from the ATHN synthase WdPks1. When combined with either no TE, its cognate WdPks1 TE, or Pks1 TE results closely paralleled those for the Pks1 parent enzyme reactions and produced $\mathrm{C}_{12}$-pyrone 7 , ATHN (5), and THN (2), respectively (Figure S2). Both WdPks1 and Pks1 produce chemically equivalent monocyclic intermediates, and their TE domains alone are responsible for the alternative synthetic outcomes.

Taken together, these results verify that Pks1 is a hexaketide synthase, but with an unprecedented bifunctional TE possessing both Claisen cyclase and deacetylase activities to release the truncated pentaketide product THN (2) directly. Pks1 is a true THN synthase and does not require a second hydrolase for polyketide shortening as has been described for melanin biosynthesis in A. fumigatus (Tsai, et al., 2001) and $W$. dermatitidis (Wheeler, et al., 2008).

\section{Incorporation of ${ }^{13} \mathrm{C}$-acetyl Starter Units}

Loss of acetyl by TE-catalyzed cleavage accounts for the absence of a "starter- unit effect" in the biosynthesis of THN from classical precursor labeling studies (Fujii, et al., 2000). To confirm that the starter unit of Pks1 was indeed acetyl, as indicated by the substrate preference of the excised Pks1 SAT domain (Crawford, et al., 2008), incorporation of $\left[1-{ }^{13} \mathrm{C}\right]$-acetate was monitored by mass spectrometry for the Pks1 TE and wA TE in vitro reactions. As expected, no mass shift was observed for flaviolin since the starter unit is removed; however, the $\mathrm{M}+1$ species indicative of labeled acetyl incorporation was observed as the major peak (M:M+1, 0.6:1) for ATHN (5), detected as 3-AF (15).

\begin{tabular}{|c|c|c|c|c|c|}
\hline Product & Peak & Observed [M+H]+ & Relative Intensity & Observed [M+Na]+ & Relative Intensity \\
\hline \multirow{3}{*}{$3-\mathrm{AF}(\mathbf{1 5})$} & $\mathrm{M}$ & 249.0390 & 0.61 & 271.0207 & 0.64 \\
\cline { 2 - 6 } & $\mathrm{M}+1$ & 250.0426 & 1 & 272.0247 & 1 \\
\hline
\end{tabular}

The unenriched products presumably contain starter units arising from decarboxylation of unlabeled malonyl units introduced by the MAT domain and may be a physiologically relevant source of chain initiators. The ability of Pks1 to access acetyl starter units from either SAT or MAT domains could improve the overall catalytic efficiency of this system. 


\section{Deacetylase Activity is Coupled to Claisen Cyclization}

ATHN (5) treated with the Pks1 TE monodomain or full length protein was not converted to THN (2), signifying that deacetylase activity was coupled to Claisen cyclization at the active site. The hexaketide pyrone 7 was also inert to ring opening and Claisen cyclization by the Pks1 TE. In separate experiments, deacetylation or deformylation of various monocyclic and bicyclic acetyl- and aldehyde-containing substrates, pictured in Figure 4, likewise failed to occur.

\section{Primary Sequence and Structural Comparisons of Melanin-Related Thioesterase Domains}

We elected to examine in greater detail what features of the Pks1 TE were important for its newly discovered, extra deacetylase function. The protein sequence of Pks1 was compared to other NR-PKSs involved in melanin and conidial pigment biosynthesis. Several YWA1producing PKSs have been functionally characterized including $A$. fumigatus Alb1(Watanabe, et al., 2000), A. nidulans wA (Watanabe, et al., 1999), A. niger FwnA (Chiang, et al., 2011; Jorgensen, et al., 2011), and Gibberella zeae Pks12 (Frandsen, et al., 2011). WdPks1 from $W$. dermatitidis is the sole validated example of an ATHN synthase (Wheeler, et al., 2008). The present study, corroborated in earlier work by Ebizuka and Fujii (Fujii, et al., 2000), confirms that Pks1 is a THN synthase. Other PKSs included in the sequence analysis have been linked to pigment biosynthesis through gene disruption, heterologous expression, or their presence in biosynthetic gene clusters with confirmed melanin pathway enzymes. The direct products of these PKSs are largely undefined; however, phylogenetic analysis demonstrates that TEs of the same known function group within a clade (Figure 5A). Sequences labeled by an asterisk were modified to reflect revised mRNA splicing based on in silico FGNESH predictions, which yielded proteins more similar to melanin-producing NR-PKSs than their current annotation. The proposed protein sequence corrections are provided in Figure S3 and result in minor changes to their C-termini.

A homology model of Pks1 TE was prepared using the recently determined $1.7 \AA$ crystal structure of the PksA TE domain (PDB 3ILS) as the template, which shares 37\% sequence identity and 53\% sequence similarity with the Pks1 TE (Figure 5B) (Korman, et al., 2010). In comparison to Pks1, the PksA TE accepts a bicyclic $\mathrm{C}_{20}$ substrate with a hexyl starterunit side chain and catalyzes an analogous Claisen cyclization to close the third ring of norsolorinic acid anthrone (Crawford, et al., 2008). The TEs adopt an $\alpha / \beta$-hydrolase fold (Nardini and Dijkstra, 1999) with a flexible lid insert between $\beta 6$ and $\beta 7$ of the canonical structure comprised of a helix-loop-helix motif that caps an internal binding chamber. Following transesterification of the PT-cyclized intermediate from ACP to TE, lid closure is thought to facilitate exclusion of water and side-chain localization to favor $\mathrm{C}-\mathrm{C}$ bond formation catalyzed by a Ser-His-Asp catalytic triad (Korman, et al., 2010). Several lid residues line the roof of the PksA TE active site and are indicated by blue triangles in the alignment of this region depicted in Figure 5C. Owing to high sequence and predicted secondary structure similarity among NR-PKSs, the lid is almost certainly a conserved feature of this sub-family of TEs. The full-length domain alignment of PksA with melanintype TEs is provided in Figure S3.

\section{Lid Swap Chimera Alter TE Activity}

Of the residues predicted to line the TE active site, the lid was identified as a potential region of sequence divergence between YWA1/ATHN- and THN-producing PKSs. Furthermore, other $\alpha / \beta$-hydrolase family members have proven amenable to lid variations and in some cases demonstrated altered substrate preferences or reactivities (Chen, et al., 2009). Therefore, a series of Pks1 and wA lid chimeras was developed by swapping the alternative lid sequences from the wA (YWA1-type), WdPks1 (ATHN-type), and Pks1 
(THN-type) proteins. Three-part Pks1 SAT-KS-MAT + PT-ACP-ACP + TE reactions accumulated THN in comparable yields to the intact full-length protein. Since the smaller proteins are expressed in greater yields, these fragments were used to assay the mutant TEs. Acetyl and malonyl $\mathrm{N}$-acetyl cysteamine (SNAC) thioesters were used as effective alternative substrates to acyl-CoA monomers. Reactions were monitored by UV-vis spectrophotometric scanning kinetics for detection of chromophore development and by HPLC for profiling product distribution. Reduction in deacetylase activity was demonstrated by diminished production of THN (2) in favor of ATHN (5). Decline in Claisen cyclase activity was indicated by accumulation of the $\mathrm{C}_{12}$-pyrone 7 . Other metabolites were not observed above trace amounts. Product distributions of the lid chimera are summarized in Figure 5D and represent the average of duplicate reactions. THN (2), which elutes as a broad peak around $14 \mathrm{~min}$, and its oxidation product flaviolin (3) were summed to give the total deacetylated product. Likewise, peak areas for ATHN (5) and oxidation product 3-AF (15) were combined.

Swapping the lid loop (LL) regions among different classes of TEs had no significant effect on either the kinetics of chromophore formation or product distribution. The loop sequence of Pks1 is four residues longer than either wA or WdPks1 but was ruled out here as a factor in deacetylation catalysis. Inclusion of the LL-flanking aL1 and aL2 helices in the lid chimeras caused a major shift in the distribution of products. Within the Pks1 parent, the WdPks1 lid efficiently complemented Claisen cyclase activity, but showed a 78\% reduction in THN relative to wild type with a concomitant increase in ATHN. The Pks1 lid-wA variant displayed reduced Claisen efficiency (pyrone was the favored product at $82 \%$ total peak area) and complete side chain retention (with no THN detected). The inverse experiment, where the Pks1 lid was integrated into the wA parent, was insufficient to turn on deacetylase activity and produced virtually no Claisen products, releasing the pyrone almost exclusively at $98 \%$ of the total.

The Pks1 lid-Wd chimera was inspected more closely due to its reduced ability to catalyze deacetylation without any apparent loss in Claisen cyclization. The aL1 and aL2 helices were individually mutated to the WdPks1 sequence (boxed residues in Figure 4C), resulting in changes to 6 amino acids per helix. Product profiles reflect the importance of aL1 for efficient removal of the acetyl side chain, which had a distribution virtually identical to the complete lid swap, whereas aL2 produced mainly THN (88\%).

Next, the hexaketide pathway homolog TE from WdPks1 was examined to see if side chain removal could be activated in a closer functional relative. Surprisingly, some THN was innately produced by the wild-type WdPks1 TE, accounting for $13 \%$ of the product output. Substitution with the Pks1 lid enhanced this basal deacetylase activity. Even though significant product was siphoned into pyrone derailment, the THN peak area increased 2fold over native Wd levels, and the ratio of Claisen products, THN:ATHN, shifted from 1:6 to $2: 1$ for this variant.

Complementation of Claisen cyclization between wA (YWA1-type) and WdPks1 (ATHNtype) TEs was also considered. The wA lid catalyzed $\mathrm{C}-\mathrm{C}$ bond formation in the WdPks1 TE with a marginal increase in the shunt $\mathrm{O}-\mathrm{C}$ population. The $\mathrm{Wd}$ lid partially complemented cyclase function in the wA TE, releasing a mixture of ATHN (34\%) and pyrone $(65 \%)$.

In some cases generation of the pyrone by TE lid chimera reactions was much greater than either no TE or Pks1 TE S2009A loss-of-function mutant controls, where transesterification of the monocyclic intermediate from ACP to TE cannot occur. Spontaneous release of this intermediate from the ACP was extremely slow and the enzyme likely wields a protective 
role in slowing the favorable $\mathrm{O}-\mathrm{C}$ cyclization mode. In contrast, the wA lid-Pks1 chimera, for example-which was deficient in Claisen cyclase activity but could presumably still accept the monocyclic substrate—accumulated much higher amounts of the pyrone. The foundation for this observed phenomenon could be passive, that is, through reduced protection of the monocyclic intermediate while covalently bound to Claisen-deficient TEs, or active, through TE-conferred catalytic release of the pyrone. Our experiments did not differentiate between these alternatives.

\section{Discussion}

No additional catalytic machinery is necessarily required to cleave the acetyl pendant by Pks1 TE. Whereas the discrete serine hydrolases from A. fumigatus (Ayg1p) and $W$. dermatitidis (WdYg1p) melanin pathways can act on YWA1 and ATHN, respectively, Pks1 TE showed no conversion of any free substrate tested for side chain removal. Lack of activity toward synthetic ATHN implies that TE acts on the immediate product of the Claisen cyclase event, perhaps prior to aromatization to the naphthalene core, where overcoming aromatic stabilization would hinder side chain hydrolysis, as depicted in Figure 6. In lieu of reprotonation to restore the resting state of the catalytic triad, the serine residue could attack into the side-chain carbonyl to release THN and acetic acid as products, analogous to $\beta$-diketide hydrolases (E.C.3.7.1.X), of which Ayg $1 \mathrm{p}$ and WdYg1p are members (Grogan, 2005). The absence of water in the active site of the closed-lid structure of PksA TE (Korman, et al., 2010) suggests that side chain attack is direct rather than water mediated by a general base mechanism in Pks1. Although speculative, we hypothesize that product partitioning between ATHN and THN is kinetically controlled, based on the fact that both Pks1 TE and WdPks1 TE synthesize both products but in highly skewed, opposing ratios. According to this model, nucleophilic attack at $\mathrm{C} 11$ would out-compete reprotonation of S2009 to shift the equilibrium of product formation toward the retro-Claisen products in Pks1. Enzyme-assisted stabilization of the pre-aromatic "diketide" intermediate may further promote this transformation.

In an alternative mechanism, deacetylation (retro-Claisen) of the TE-bound monocyclic intermediate may occur prior to Claisen cyclization (Dieckmann) with the instantly formed enolate closing directly to THN. We consider this scenario, however, less likely since a suitable base could not be identified in the homology model of the Pks1 TE in the region where the side chain is presumed to reside. Structural analyses of closely related melaninTEs might illuminate how small changes in their active site geometries could contribute to product partitioning.

Domain swapping exercises illustrate the interchangeability of NR-PKS domains among closely related synthases. The wA TE, which accepts a native $\mathrm{C}_{14}$-ACP-bound substrate, proficiently catalyzed Claisen cyclization of the Pks1-generated $\mathrm{C}_{12}$ intermediate. This promiscuity in side chain binding indicates that the precise identity of the terminal acetyl group of the YWA1 TE-bound precursor is not the sole determinant for orienting the side chain for Claisen/Dieckmann C-C bond formation in wA. Furthermore, secondary structural motifs within domains have proven adaptable to exchange and are useful in deciphering what protein features are responsible for specific activities and ultimately, natural product programming. Through generation of TE chimera, we have shown that the lid region, specifically the aL1 helix, is a factor in maintaining the active site topography of Pks1 TE necessary to catalyze efficient deacetylation, a previously unknown function for NR-PKS TE domains. Another recent example where this approach was successfully applied involved structural swaps between ketoreductase domains from resorcylic acid macrolactone synthases, members of the highly-reducing class of fungal iterative PKSs, to understand the rationale for differential stereochemistries of their product hydroxyls (Zhou, et al., 2012). 
Stepwise production of THN via an isolable acyl side-chain intermediate (YWA1 or ATHN), leads to greater potential for synthetic diversity. In A. niger, for example, FwnA produces naphtho- $\gamma$-pyrones, including YWA1, that can be directly incorporated into natural products (like the dimeric aurosperones) or, through selective use of the Ayg1p homolog OlvA, be directed into the DHN melanin pathway (Chiang, et al., 2011). Alternatively, C. lagenarium (and likely many other melanin NR-PKSs that are closely related) has a bifunctional TE domain that catalyzes both Claisen cyclization and deacetylation. Melanin is important for fungal survival and longevity, as well as virulence in many pathogenic species. Depending on the selective pressures exerted on different fungal strains, more efficient THN biosynthesis may have evolved in response to environmental factors necessitating greater proficiency in cell wall pigmentation at the expense of product diversity.

\section{Significance}

Fungal melanins provide survival advantages in harsh environments. They are also virulence factors for many pathogenic fungi where pigment disruption often renders these strains noninfective. Similar heptaketide and hexaketide pathways have been described that utilize separate deacetylases to remove the acetoacetyl and acetyl side chains of their respective bicyclic substrates released by standard C-terminal thioesterase/Claisen cyclase domains of NR-PKSs. In vitro biochemical characterization confirms that Pks1 efficiently synthesizes THN unaided by a second enzyme, defining an efficient third fungal route to this important melanin precursor. Pks1 TE is both a Claisen cyclase and deacetylase that processes an enzyme-bound monocyclic hexaketide precursor, explaining the absence of an observable "starter-unit effect" in this system. Domain and secondary structural motif swapping experiments could provide a valid route to engineering NR-PKS pathways to expand the biosynthetic potential of these enzymes and in understanding the molecular foundation of melanogenesis, where it may aid efforts to discover new, effective anti-fungal therapies. The unusual ability of Pks1 TE to catalyze tandem carbon- carbon bond making and breaking reactions in a single enzymatic step further expands the diverse array of reactions known for $\alpha / \beta$-hydrolase fold enzymes generally (Ollis and Carr, 2009), and the repertoire of activities exemplified by fungal TE domains in particular.

\section{Experimental Procedures}

\section{Synthesis of Melanin Standards}

All synthetic details are provided in the Supplemental Information.

\section{C. orbiculare cDNA Analysis}

C. orbiculare NRRL28842 was cultured in potato-sucrose liquid medium supplemented with $0.2 \%$ yeast extract and mycelia were transferred to $1.2 \mathrm{M}$ sucrose for $20 \mathrm{~h}$ as previously described to rapidly induce transcription of melanin biosynthetic pathway genes (Okamoto, et al., 2001; Takano, et al., 1995). Mycelia were harvested by vacuum filtration over Miracloth (EMD Millipore, Billerica, MA), frozen in liquid $\mathrm{N}_{2}$, and stored at $-80^{\circ} \mathrm{C}$ until use. Total RNA was harvested from mycelia ground in liquid $\mathrm{N}_{2}$ using an RNeasy Plant Mini Kit (Qiagen, Valencia, CA) as recommended by the manufacturer with the on-column DNase digestion step. First strand cDNA was prepared from $1 \mu \mathrm{g}$ total RNA and random hexamer primer (IDT, Coralville, IA) using M-MLV reverse transcriptase (Promega, Madison, WI) in a $20 \mu \mathrm{L}$ volume. A reaction lacking reverse transcriptase was used as a negative control. The cDNA synthesis reaction was used directly as template for amplification of the region spanning the proposed exon 4 in pks1 using primer set: Pks1e4check-5.1 5' -GAAGGCGAGACAGTCCAAAG -3' and Pksle4check-3.1 5' - 
TAAGAGTGTGCATGATCTCGC-3'. PCRs contained $2 \mu \mathrm{L}$ cDNA, $200 \mu \mathrm{M}$ deoxynucleoside triphosphates, $0.4 \mu \mathrm{M}$ each primer, $3 \%$ dimethyl sulfoxide, and 2.5 units OneTaq DNA Polymerase (New England BioLabs, Ipswich, MA) in manufacturer provided buffer in $50 \mu \mathrm{L}$ volume. A touchdown PCR method was employed. The thermocycler conditions were $94{ }^{\circ} \mathrm{C} 0: 15$ (melt), $60{ }^{\circ} \mathrm{C}$ to $52{ }^{\circ} \mathrm{C} \mathrm{0:30}\left(1{ }^{\circ} \mathrm{C} /\right.$ cycle incremental temperature drop, anneal), $68^{\circ} \mathrm{C} 1: 20$ (extend) for eight cycles, then annealing temperature was maintained at $52{ }^{\circ} \mathrm{C}$ for an additional 20 cycles. Total RNA and PCR amplified cDNA were analyzed by agarose gel electrophoresis. The cDNA amplicon was subcloned into pCRIITOPO using a TOPO TA Cloning Kit (Invitrogen, Grand Island, NY) and purified plasmids from four colonies were individually sequenced to confirm the revised splicing prediction. The cDNA inserts were aligned in Sequencher v4.0 (Gene Codes Corporation, Ann Arbor, $\mathrm{MI})$ and the contig is provided in the Supplemental Information.

\section{Cloning and Expression}

DNA manipulations were carried out using standard methods (Sambrook and Russell, 2001). wA and Pks1 fragments were amplified from A. nidulans RLMH67 and $C$. orbiculare (syn. C. lagenarium) NRRL 28842 gDNA, respectively. The $W$. dermatitidis TE coding sequence DNA was purchased from GenScript (Piscataway, NJ). Point mutants and lid chimeras were generated by overlap extension PCR using wild-type TE constructs as template DNA. Subsequently, additional WdPks1 fragments were amplified from $W$. dermatitidis UT8656 gDNA. Further cloning details are summarized in the Supplemental Information (Tables S2 and S3). Recombinant proteins were heterologously expressed from BL21(DE3) in LuriaBertani broth medium and purified by $\mathrm{Ni}^{2+}$-affinity chromatography as previously described using standard methods (Crawford, et al., 2008).

\section{In Vitro Reactions}

Reactions were carried out at room temperature with $10 \mu \mathrm{M}$ protein (each) in reaction buffer [100 mM K/PO 4 pH 7.0, $1 \mathrm{mM}$ tris(2-carboxyethyl)phosphine (TCEP)] and were initiated by addition of substrate mix containing acetyl and malonyl as the CoA or SNAC thioesters. Reactions were monitored by UV-vis spectrophotometry using scanning kinetics from 280 $650 \mathrm{~nm}$ on a Cary 50 instrument (Varian Inc., Walnut Creek, CA). Products were extracted into ethyl acetate or filtered through a 10k MWCO Microcon centrifugal filter device (Millipore, Billerica, MA) for HPLC analysis. Products (100 $\mu \mathrm{L}$ injections) were separated by reverse phase HPLC (Agilent 1200, Santa Clara, CA) using a bisolvent linear gradient over a Prodigy ODS3 column $(4.6 \times 250 \mathrm{~mm}, 5 \mu \mathrm{m}$, Phenomenex, Torrence, CA $)$. Solvent A: $\mathrm{H}_{2} \mathrm{O}+0.1 \%$ formic acid, Solvent B: acetonitrile $+0.1 \%$ formic acid; 5 to $80 \%$ B over 30 min with a flow rate of $1 \mathrm{~mL} / \mathrm{min}$.

In initial TE swaps, Pks1 $\triangle$ TE was combined with either no TE, Pks1 TE, or wA TE with $0.5 \mathrm{mM}$ acetyl-CoA and $2 \mathrm{mM}$ malonyl-CoA substrates. After $4 \mathrm{~h}$, reactions were filtered through Microcon centrifugal filter devices and analyzed by HPLC. Full length Pks1 and three part (SAT-KS-MAT + PT-ACP-ACP + TE) combinations were likewise tested.

Wild-type, point-mutant, and lid-chimera TEs were assayed in three-part multidomain combinations with Pks1 SAT-KS-MAT and PT-ACP-ACP using $0.25 \mathrm{mM}$ acetyl-SNAC and $1 \mathrm{mM}$ malonyl-SNAC substrates in reaction buffer additionally containing $10 \%$ glycerol. Reactions were monitored by UV-vis spectrophotometry for $2 \mathrm{~h}$ and filtered through Microcon centrifugal filter devices. Products were separated by HPLC and the resulting chromatograms $\left(\mathrm{A}_{254} \mathrm{~nm}\right)$ integrated to calculate peak areas. Control reactions with WdPks1 SAT-KS-MAT and PT-ACP-ACP were similarly conducted. 


\section{Deacetylase/Deformylase Assay}

ATHN (5), $\mathrm{C}_{12}$-pyrone 7, and a variety of other acetyl and aldehyde side-chain compounds (Figure 5, $0.5 \mathrm{mM}$ ) were separately incubated with the Pks1 TE or S2009A mutant control $(10 \mu \mathrm{M})$ overnight. Reactions were filtered through Microcon centrifugal filter devices and analyzed by HPLC. ATHN (5) was also tested as a substrate for full length Pks1.

\section{Starter Unit Incorporation Assay}

Three-part in vitro reactions containing Pks1 SAT-KS-MAT, Pks1 PT-ACP-ACP, and either Pks1 TE or wA TE were initiated with $1 \mathrm{mM}\left[1-{ }^{13} \mathrm{C}\right]$-acetyl-SNAC and $1 \mathrm{mM}$ malonyl-SNAC. Following overnight incubation, $500 \mu \mathrm{L}$ reactions were quenched with 10 $\mu \mathrm{L} \mathrm{HCl}$ and extracted twice with $1 \mathrm{~mL}$ ethyl acetate. Pooled extracts were evaporated to dryness and dissolved in $250 \mu \mathrm{L} \mathrm{50 \%}$ aqueous acetonitrile. Products were analyzed by HRESI-LCMS on a Shimadzu LC-IT-TOF over a Luna C18(2) analytical column $(2.0 \times 150$ $\mathrm{mm}, 3 \mu \mathrm{m}$, Phenomenex) using a linear gradient of 20 to $80 \%$ B over 30 min with a flow rate of $0.2 \mathrm{~mL} / \mathrm{min}$. Positive ion peak intensities were compared for the $\mathrm{M}$ and $\mathrm{M}+1$ peaks using LCMS Solutions v3.50 software (Shimadzu, Columbia, MD).

\section{Homology Model of Pks1 TE}

A homology model for the Pks1 TE domain was generated with the loopmodel algorithm of MODELER within the Accelrys Discovery Studio Suite v2.1 (Accelrys Inc., San Diego, CA). The PksA TE domain (PDB 3ILS) was set as the template structure. The model was built based on ClustalX alignments of 37 NR-PKS TE sequences, and overhangs were cut from the sequences.

\section{Sequence Analyses}

A list of validated NR-PKSs involved in melanin and conidial pigment biosynthesis was compiled (Table S1). Analyses were conducted in MEGA5 (Tamura, et al., 2011) and involved 29 proteins. Sequence alignment was completed with ClustalX2 using default settings. The gDNA from potentially mismatched protein sequences was submitted to FGNESH gene prediction software (softberry.com) to consider alterations to splicing patterns. In cases where better sequence similarity to other NR-PKSs was achieved by revised splicing, these sequences were included in lieu of the current annotation. The evolutionary history was inferred using the neighbor-joining method and a bootstrap consensus tree was composed from 1000 replicates. All ambiguous positions were removed for each sequence pair. Evolutionary distances were computed using the Poisson correction method.

\section{Supplementary Material}

Refer to Web version on PubMed Central for supplementary material.

\section{Acknowledgments}

We thank Prof. J.M. Crawford (Yale University, New Haven, CT) for preliminary DNA work and helpful discussions. Drs. S.A. Rehner (USDA-ARS, Beltsville, MD) and K. O'Donnell (USDA, Peoria, IL), Prof. N. P. Keller (Univ. of Wisconsin, Madison, WI), and Dr. Z. Wang (Naval Research Laboratory, Washington, DC) and Prof. P.J. Szaniszlo (Univ. of Texas, Austin, TX) kindly provided spores and/or gDNA from C. orbiculare, $A$. nidulans, and $W$. dermatitidis, respectively. We are grateful to Katherine Belecki for collecting mass spectrometry data and to K.L. Fiedler and Prof. R.J. Cotter of the Middle Atlantic Mass Spectrometry Laboratory at the Johns Hopkins School of Medicine and the Mid-Atlantic Regional Office of Shimadzu Scientific Instruments, Inc. (Columbia, MD) for use of their Shimadzu LC-IT-TOF. This work was supported by the National Institutes of Health Grant ES 001670. 


\section{References}

Akao T, Sano M, Yamada O, Akeno T, Fujii K, Goto K, Ohashi-Kunihiro S, Takase K, YasukawaWatanabe M, Yamaguchi K, et al. Analysis of expressed sequence tags from the fungus Aspergillus oryzae cultured under different conditions. DNA Research. 2007; 14:47-57. [PubMed: 17540709]

Bardshiri E, Simpson TJ. 13C and 2H labelling studies on the biosynthesis of scytalone in Phialaphora lagerbergii. Tetrahedron. 1983; 39:3539-3542.

Bell AA, Wheeler MH. Biosynthesis and Functions of Fungal Melanins. Annu Rev Phytopathol. 1986; 24:411-451.

Chen B, Cai Z, Wu W, Huang Y, Pleiss J, Lin Z. Morphing activity between structurally similar enzymes: from heme-free bromoperoxidase to lipase. Biochemistry. 2009; 48:11496-11504. [PubMed: 19883129]

Chiang Y-M, Meyer KM, Praseuth M, Baker SE, Bruno KS, Wang CC. Characterization of a polyketide synthase in Aspergillus niger whose product is a precursor for both dihydroxynaphthalene (DHN) melanin and naphtho-[gamma]-pyrone. Fungal Genet Biol. 2011; 48:430-437. [PubMed: 21176790]

Crawford JM, Dancy BCR, Hill EA, Udwary DW, Townsend CA. Identification of a starter unit acylcarrier protein transacylase domain in an iterative type I polyketide synthase. Proc Natl Acad Sci U S A. 2006; 103:16728-16733. [PubMed: 17071746]

Crawford JM, Korman TP, Labonte JW, Vagstad AL, Hill EA, Kamari-Bidkorpeh O, Tsai SC, Townsend CA. Structural basis for biosynthetic programming of fungal aromatic polyketide cyclization. Nature. 2009; 461:1139-1143. [PubMed: 19847268]

Crawford JM, Thomas PM, Scheerer JR, Vagstad AL, Kelleher NL, Townsend CA. Deconstruction of iterative multidomain polyketide synthase function. Science. 2008; 320:243-246. [PubMed: 18403714]

Crawford JM, Townsend CA. New insights into the formation of fungal aromatic polyketides. Nat Rev Microbiol. 2010; 8:879-889. [PubMed: 21079635]

Crawford JM, Vagstad AL, Whitworth KP, Ehrlich KC, Townsend CA. Synthetic strategy of nonreducing iterative polyketide synthases and the origin of the classical "starter-unit effect". ChemBioChem. 2008; 9:1019-1023. [PubMed: 18338425]

Cunha M, Franzen A, Seabra S, Herbst M, Vugman N, Borba L, de Souza W, Rozental S. Melanin in Fonsecaea pedrosoi: a trap for oxidative radicals. BMC Microbiol. 2010; 10

Frandsen RJN, Schutt C, Lund BW, Staerk D, Nielsen J, Olsson S, Giese H. Two novel classes of enzymes are required for the biosynthesis of aurofusarin in Fusarium graminearum. J Biol Chem. 2011; 286:10419-10428. [PubMed: 21296881]

Fujii I, Mori Y, Watanabe A, Kubo Y, Tsuji G, Ebizuka Y. Enzymatic synthesis of 1,3,6,8tetrahydroxynaphthalene solely from malonyl coenzyme A by a fungal iterative type I polyketide synthase PKS1. Biochemistry. 2000; 39:8853-8858. [PubMed: 10913297]

Fujii I, Mori Y, Watanabe A, Kubo Y, Tsuji G, Ebizuka Y. Heterologous expression and product identification of Colletotrichum lagenarium polyketide synthase encoded by the PKS1 gene involved in melanin biosynthesis. Biosci Biotechnol Biochem. 1999; 63:1445-1452. [PubMed: 10501004]

Fujii I, Watanabe A, Sankawa U, Ebizuka Y. Identification of Claisen cyclase domain in fungal polyketide synthase WA, a naphthopyrone synthase of Aspergillus nidulans. Chem Biol. 2001; 8:189-197. [PubMed: 11251292]

Funa N, Funabashi M, Ohnishi Y, Horinouchi S. Biosynthesis of hexahydroxyperylenequinone melanin via oxidative aryl coupling by cytochrome P-450 in Streptomyces griseus. J Bacteriol. 2005; 187:8149-8155. [PubMed: 16291687]

Funa N, Ohnishi Y, Ebizuka Y, Horinouchi S. Properties and substrate specificity of RppA, a chalcone synthase-related polyketide synthase in Streptomyces griseus. J Biol Chem. 2002; 277:4628-4635. [PubMed: 11723138]

Gladieux, P.; Byrnes, EJ., III; Aguileta, G.; Fisher, CM.; Heitman, J.; Giraud, T. Epidemiology and evolution of fungal pathogens in plants and animals. In: Tibayrenc, M., editor. Genetics and Evolution of Infectious Disease. London: Elsevier; 2011. p. 59-132. 
Grogan G. Emergent mechanistic diversity of enzyme-catalysed beta-diketone cleavage. Biochemical Journal. 2005; 388:721-730. [PubMed: 15934927]

Jacobson ES. Pathogenic Roles for Fungal Melanins. Clin Microbiol Rev. 2000; 13:708-717. [PubMed: 11023965]

Jorgensen TR, Park J, Arentshorst M, van Welzen AM, Lamers G, vanKuyk PA, Damveld RA, van den Hondel CAM, Nielsen KF, Frisvad JC, et al. The molecular and genetic basis of conidial pigmentation in Aspergillus niger. Fungal Genet Biol. 2011; 48:544-553. [PubMed: 21277986]

Korman TP, Crawford JM, Labonte JW, Newman AG, Wong J, Townsend CA, Tsai S-C. Structure and function of an iterative polyketide synthase thioesterase domain catalyzing Claisen cyclization in aflatoxin biosynthesis. Proc Natl Acad Sci U S A. 2010; 107:6246-6251. [PubMed: 20332208]

Kubo Y, Suzuki K, Furusawa I, Yamamoto M. Melanin biosynthesis as a prerequisite for penetration by appressoria of Colletotrichum lagenarium: Site of inhibition by melanin-inhibiting fungicides and their action on appressoria. Pestic Biochem Physiol. 1985; 23:47-55.

Langfelder K, Streibel M, Jahn B, Haase G, Brakhage A. Biosynthesis of fungal melanins and their importance for human pathogenic fungi. Fungal Genet Biol. 2003; 38:143-158. [PubMed: 12620252]

Li Y, Xu W, Tang Y. Classification, prediction and verification of the regioselectivity of fungal polyketide synthase product template domains. J Biol Chem. 2010; 285:22764-22773. [PubMed: 20479000]

Liu GY, Nizet V. Color me bad: microbial pigments as virulence factors. Trends Microbiol. 2009; 17:406-413. [PubMed: 19726196]

Machida M, Asai K, Sano M, Tanaka T, Kumagai T, Terai G, Kusumoto KI, Arima T, Akita O, Kashiwagi Y, et al. Genome sequencing and analysis of Aspergillus oryzae. Nature. 2005; 438:1157-1161. [PubMed: 16372010]

Nardini M, Dijkstra BW. $\alpha / \beta$ Hydrolase fold enzymes: the family keeps growing. Curr Opin Struct Biol. 1999; 9:732-737. [PubMed: 10607665]

Nosanchuk JD, Casadevall A. Impact of melanin on microbial virulence and clinical resistance to antimicrobial compounds. Antimicrob Agents Chemother. 2006; 50:3519-3528. [PubMed: 17065617]

Okamoto S, Sakurada M, Kubo Y, Tsuji G, Fujii I, Ebizuka Y, Ono M, Nagasawa H, Sakuda S. Inhibitory effect of aflastatin A on melanin biosynthesis by Colletotrichum lagenarium. Microbiology. 2001; 147:2623-2628. [PubMed: 11535802]

Ollis DL, Carr PD. a/ $\beta$ Hydrolase fold: an update. Protein Pept Lett. 2009; 16:1137-1148. [PubMed: 19508187]

Pihet M, Vandeputte P, Tronchin G, Renier G, Saulnier P, Georgeault S, Mallet R, Chabasse D, Symoens F, Bouchara J-P. Melanin is an essential component for the integrity of the cell wall of Aspergillus fumigatus conidia. BMC Microbiol. 2009; 9

Sambrook, J.; Russell, DW. Molecular cloning: a laboratory manual. Cold Spring Harbor Laboratory Press; 2001.

Takano Y, Kubo Y, Kawamura C, Tsuge T, Furusawa I. The Alternaria alternata melanin biosynthesis gene restores appressorial melanization and penetration of cellulose membranes in the melanindeficient albino mutant of Colletotrichum lagenarium. Fungal Genet Biol. 1997; 21:131-140.

Takano Y, Kubo Y, Shimizu K, Mise K, Okuno T, Furusawa I. Structural analysis of PKS1, a polyketide synthase gene involved in melanin biosynthesis in Colletotrichum lagenarium. Mol. Genet. Genomics. 1995; 249:162-167.

Tamura K, Peterson D, Peterson N, Stecher G, Nei M, Kumar S. MEGA5: molecular evolutionary genetics analysis using maximum likelihood, evolutionary distance, and maximum parsimony methods. Mol Biol Evol. 2011; 28:2731-2739. [PubMed: 21546353]

Thywißen A, Heinekamp T, Dahse H-M, Schmaler-Ripcke J, Nietsche S, Zipfel PF, Brakhage AA. Conidial dihydroxynaphthalene melanin of the human pathogenic fungus Aspergillus fumigatus interferes with the host endocytosis pathway. Front Microbiol. 2011; 2

Tsai HF, Fujii I, Watanabe A, Wheeler MH, Chang YC, Yasuoka Y, Ebizuka Y, Kwon-Chung KJ. Pentaketide melanin biosynthesis in Aspergillus fumigatus requires chain-length shortening of a heptaketide precursor. J Biol Chem. 2001; 276:29292-29298. [PubMed: 11350964] 
Tsuji G, Tsuge S, Shiraishi T, Kubo Y. Expression pattern of melanin biosynthesis enzymes during infectious morphogenesis of Colletotrichum lagenarium. J Gen Plant Pathol. 2003; 69:169-175.

Udwary DW, Merski M, Townsend CA. A method for prediction of the locations of linker regions within large multifunctional proteins, and application to a type I polyketide synthase. J Mol Biol. 2002; 323:585-598. [PubMed: 12381311]

Ushimaru T, Terada H, Tsuboi K, Kogou Y, Sakaguchi A, Tsuji G, Kubo Y. Development of an efficient gene targeting system in Colletotrichum higginsianum using a non-homologous endjoining mutant and Agrobacterium tumefaciens-mediated gene transfer. Mol. Genet. Genomics. 2010; 284:357-371.

Watanabe A, Ebizuka Y. A novel hexaketide naphthalene synthesized by a chimeric polyketide synthase composed of fungal pentaketide and heptaketide synthases. Tetrahedron Lett. 2002; 43:843-846.

Watanabe A, Fujii I, Sankawa U, Mayorga ME, Timberlake WE, Ebizuka Y. Reidentification of Aspergillus nidulans wA gene to code for a polyketide synthase of naphthopyrone. Tetrahedron Lett. 1999; 40:91-94.

Watanabe A, Fujii I, Tsai HF, Chang YC, Kwon-Chung KJ, Ebizuka Y. Aspergillus fumigatus alb1 encodes naphthopyrone synthase when expressed in Aspergillus oryzae. FEMS Microbiol Lett. 2000; 192:39-44. [PubMed: 11040426]

Wheeler MH, Abramczyk D, Puckhaber LS, Naruse M, Ebizuka Y, Fujii I, Szaniszlo PJ. New biosynthetic step in the melanin pathway of Wangiella (Exophiala) dermatitidis: evidence for 2acetyl-1,3,6,8-tetrahydroxynaphthalene as a novel precursor. Eukaryot Cell. 2008; 7:1699-1711. [PubMed: 18676950]

Wheeler MH, Klich MA. The effects of tricyclazole, pyroquilon, phthalide, and related fungicides on the production of conidial wall pigments by Penicillium and Aspergillus species. Pestic Biochem Physiol. 1995; 52:125-136.

Woo PCY, Tam EWT, Chong KTK, Cai JJ, Tung ETK, Ngan AHY, Lau SKP, Yuen K-Y. High diversity of polyketide synthase genes and the melanin biosynthesis gene cluster in Penicillium marneffei. FEBS J. 2010; 277:3750-3758. [PubMed: 20718860]

Zhang W, Li Y, Tang Y. Engineered biosynthesis of bacterial aromatic polyketides in Escherichia coli. Proc Natl Acad Sci U S A. 2008; 105:20683-20688. [PubMed: 19075227]

Zhou H, Gao Z, Qiao K, Wang J, Vederas JC, Tang Y. A fungal ketoreductase domain that displays substrate-dependent stereospecificity. Nat Chem Biol. 2012; 8:331-333. [PubMed: 22406519]

Chem Biol. Author manuscript; available in PMC 2013 December 21. 


\section{Highlights}

- Revised C. lagenarium Pks1 coding sequence enabling in vitro analysis

- Clarified third fungal route to dihydroxynaphthalene melanin

- Revealed thioesterase-catalyzed tandem Claisen cyclization and deacetylation

- Lid swap chimera illuminate features necessary for $\mathrm{C}-\mathrm{C}$ bond making and breaking 


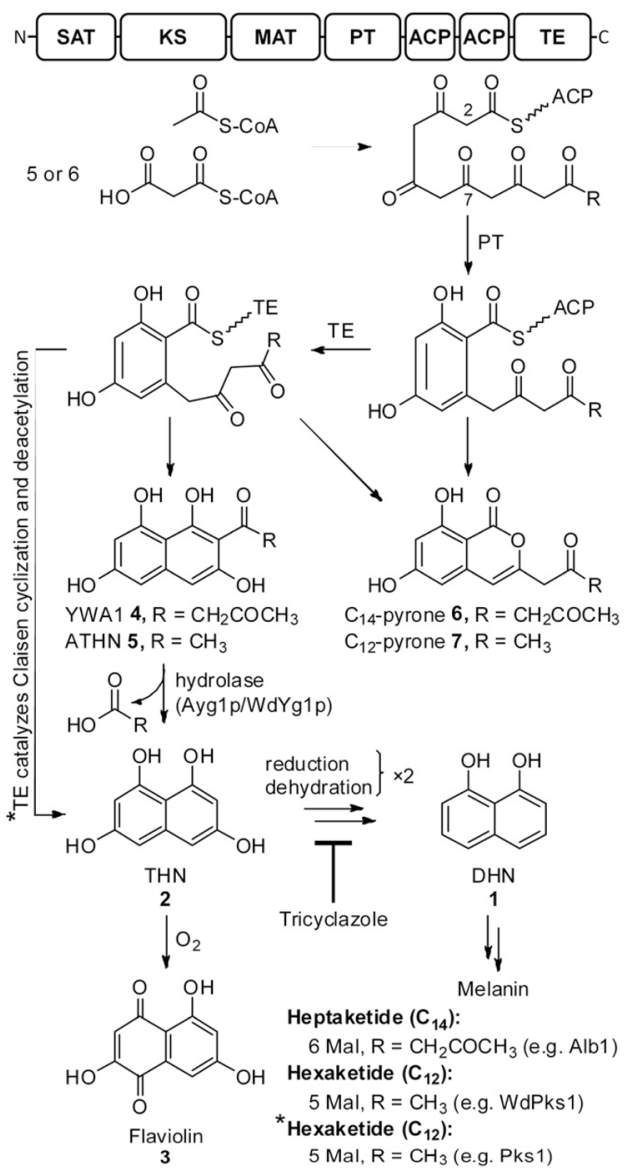

Figure 1.

Convergent biosynthetic routes to the THN (2) intermediate in fungal DHN (1) melanin biosynthesis by NR-PKSs with tandem ACP domains. Pyrones (6 and 7) are released from heptaketide and hexaketide pathways as shunt metabolites. Pathways blocked at the THN reductase, for example, by treatment with tricyclazole, accumulate flaviolin $(3)$, the autoxidation product of 2 . 


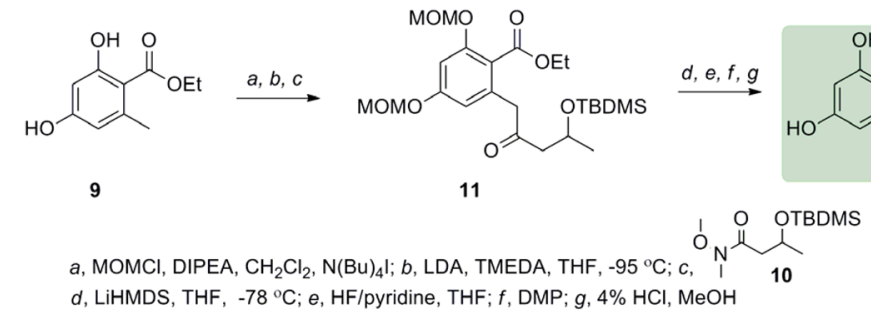

B
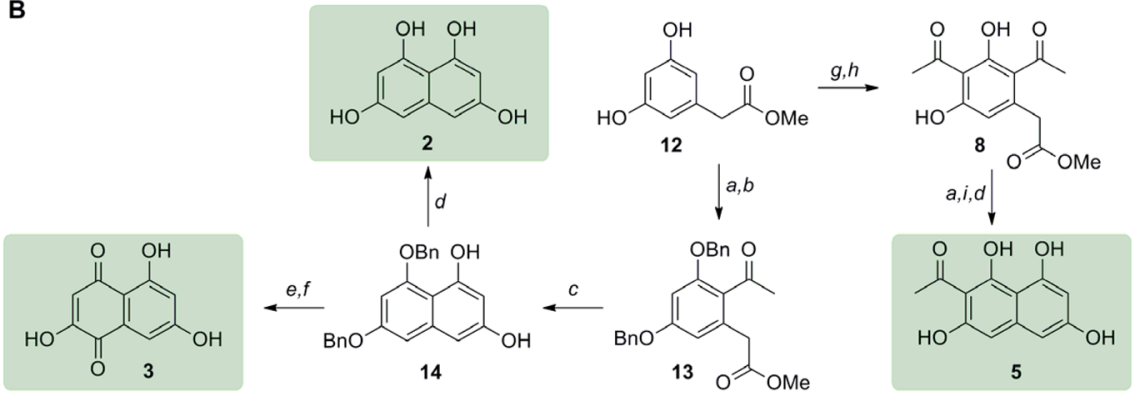

a, $\mathrm{BnBr}, \mathrm{K}_{2} \mathrm{CO}_{3}$, acetone, reflux; $b, \mathrm{Ac}_{2} \mathrm{O}, \mathrm{HClO}_{4} ; c, \mathrm{NaOMe}, \mathrm{MeOH} ; d, \mathrm{Pd} / \mathrm{C}, \mathrm{H}$, $\mathrm{EtOH}, \mathrm{AcOH} ; e, \mathrm{Ac}_{2} \mathrm{O}, \mathrm{NaOAc}, \mathrm{Pd} / \mathrm{C}$; $f, \mathrm{~K}_{2} \mathrm{CO}_{3}, \mathrm{EtOH}$, air; $g, \mathrm{Ac}_{2} \mathrm{O}$, pyridine; $h, \mathrm{AlCl}_{3}, \mathrm{DCE}$, reflux; $i$, LiHMDS, THF $0{ }^{\circ} \mathrm{C}$.

Figure 2.

Overview of syntheses for melanin standards. A) Synthesis of $\mathrm{C}_{12}$-pyrone 7. B) Synthesis of THN (2), flaviolin (3), and ATHN (5). See also Figure S1 for HPLC separation of standards. 


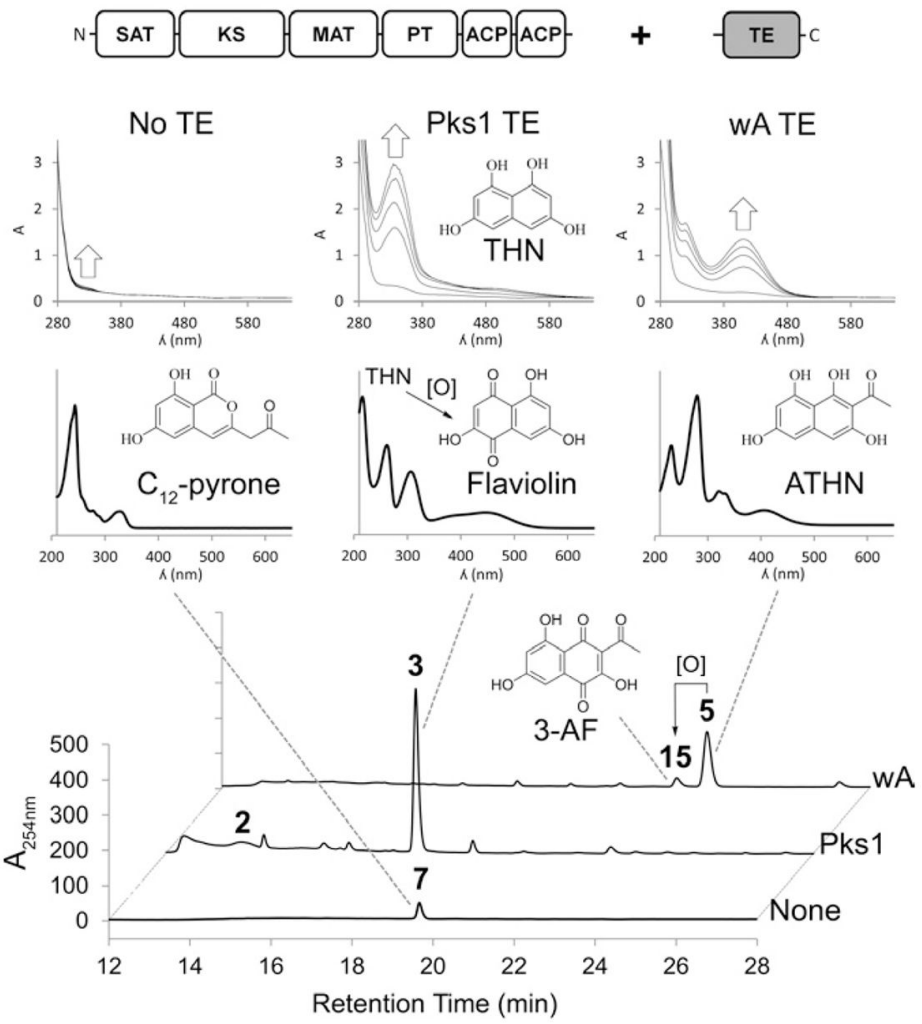

Figure 3.

Two-part multidomain combination TE assay: Pks1 $\Delta$ TE reacted with either no, Pks1, or wA TE. Reactions were monitored by UV-vis spectrophotometry scanning every $10 \mathrm{~min}$ from 280-650 nm. Products were separated by HPLC using a linear gradient. THN (2) initially accumulates in the Pks1 TE reaction, indicated by increasing absorbance at $336 \mathrm{~nm}$, and then spontaneously oxidizes to flaviolin (3), the major product detected by HPLC. ATHN (5) likewise spontaneously oxidizes to 3-AF (15). Diode array spectra were extracted for the major product peak from each HPLC chromatogram. See also Figure S2 for WdPks1 parent TE swap control reactions. 
<smiles>CC(=O)/C=C(\O)c1ccc(O)cc1O</smiles><smiles>CC(=O)c1c(O)c2ccccc2oc1=O</smiles><smiles>CC(=O)C1CCc2ccccc2C1=O</smiles><smiles>COc1cc(O)c(C(C)=O)c(OC)c1</smiles><smiles>CC(=O)c1ccc(O)cc1O</smiles><smiles>COc1cc(O)cc(O)c1C=O</smiles><smiles>O=Cc1c(O)cc(O)cc1O</smiles>

Figure 4.

Additional substrates tested for side chain removal by the Pks1 TE monodomain. No conversion was detected for any compound. 
A

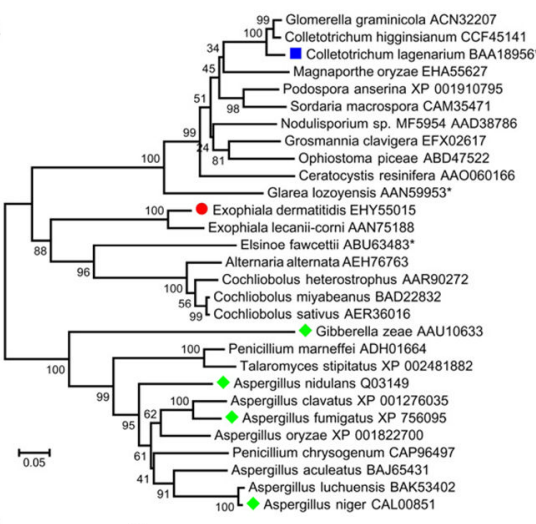

D

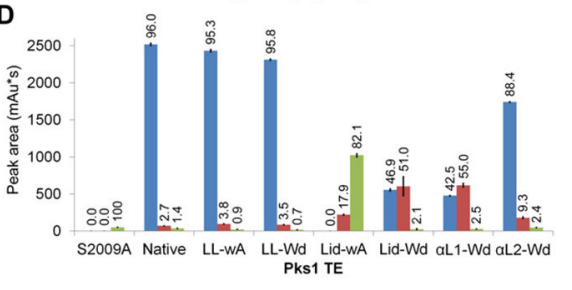

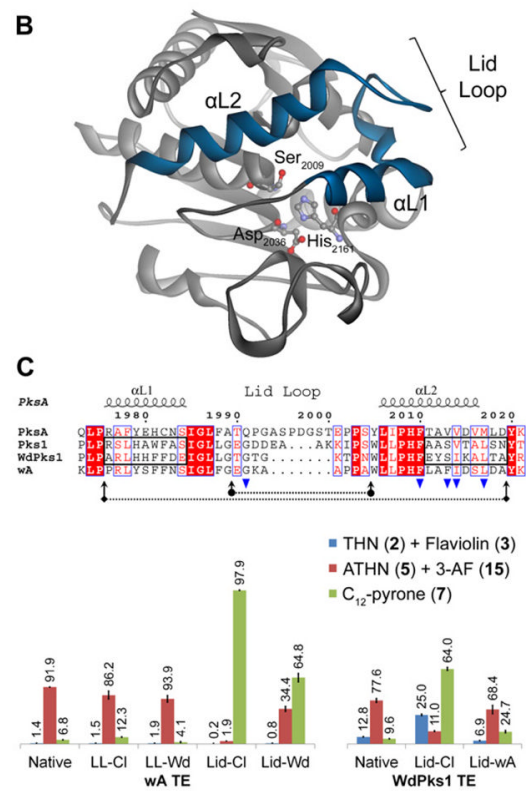

Figure 5.

Comparative analyses of TE domains. A) Phylogenetic tree of NR-PKSs with confirmed involvement in melanin or conidial pigment biosynthesis. The direct product of the PKS, when known, is indicated by either a blue square $(=$ THN), red circle $(=$ ATHN), or green diamond (= YWA1). Bootstrap values are shown next to the branch points. See also Table S1. B) Homology model of Pks1 TE based on the PksA TE structure PDB ILS3. The lid region used in chimera swaps is colored blue and the catalytic triad is shown as balls and sticks colored by element. C) Sequence alignment of the lid region with PksA numbering. Hydrophobic residues lining the PksA active site are indicated by blue triangles. Arrows indicate lid loop (circle) and complete lid (diamond) regions swapped in the chimeras. The black boxes surrounding Pks1 and $\mathrm{WdPks} 1$ residues indicate the regions mutated in helix swaps. See also Figure S3 for the full TE sequence alignment. D) Product distribution of three-part TE assay reactions organized by parent TE. Labels indicate \% peak area for each product. Error bars show standard deviation from duplicate reactions. LL $=$ Lid loop, $\mathrm{L}=$ $\mathrm{Lid} ; \mathrm{Cl}=C$. lagenarium $\mathrm{Pks} 1, \mathrm{wA}=A$. nidulans $\mathrm{wA}$, and $\mathrm{Wd}=W$. dermatitidis $\mathrm{WdPks} 1$. 


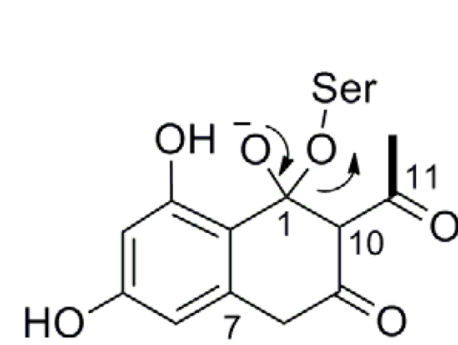<smiles>CC(=O)C1C(=O)Cc2cc(O)cc(O)c2C1=O</smiles><smiles>O[SeH]</smiles><smiles>CCC</smiles><smiles>[Y][IH+]1CC(=O)C(C(C)=O)=C([O-])c2c(O)cc(O)cc21</smiles>

ATHN 5<smiles>[Y][CH+]Cc1cc(O)cc(O)c1C([O-])=CC(=O)O[Se]OC(C)=O</smiles>

THN 2

Figure 6.

Proposed mechanisms for product partitioning in WdPks1 and Pks1 TEs, which synthesize ATHN (5) and THN (2), respectively. The acetyl starter unit is shown in bold. 